\title{
Elongational Effect on Immiscible Polymer Blends via Novel Vane Plasticating Extruder
}

\author{
Zhenghuan WU*, Quan WANG**, Yongqing ZHAO***, Qixiang FAN****, Hai YANG*****, \\ Jinping QU******* \\ *College of mechanical engineering, Tianjin Key Laboratory of High Speed Cutting and Precision Machining, Tianjin \\ University of Technology and Education, Tianjin 300222, China, E-mail: wzh46817@163.com; \\ **College of mechanical engineering, Tianjin Key Laboratory of High Speed Cutting and Precision Machining, Tianjin \\ University of Technology and Education, Tianjin 300222, China, E-mail: wq2001126@163.com \\ ***National Engineering Research Center of Novel Equipment for Polymer Processing, the Key Laboratory of Polymer \\ Processing Engineering of Ministry of Education, South China University of Technology, Guangzhou 510640, Guang- \\ dong, China, E-mail: zhaoyongqing389@163.com \\ ****College of mechanical engineering, Tianjin Key Laboratory of High Speed Cutting and Precision Machining, Tianjin \\ University of Technology and Education, Tianjin 300222, China, E-mail:_qxfan2015@163.com \\ *****Guangdong Institute of Automation, Equipment of Automation, Guangzhou 510640, Guangdong, China, E-mail: \\ 276840530@qq.com \\ ******National Engineering Research Center of Novel Equipment for Polymer Processing, the Key Laboratory of Poly- \\ mer Processing Engineering of Ministry of Education, South China University of Technology, Guangzhou 510640, \\ Guangdong, China, E-mail: jpqu@scut.edu.cn
}

crossref http://dx.doi.org/10.5755/j01.mech.23.6.19855

\section{Nomenclature}

$V_{P E}$ - fibrils volume of LLDPE in PS matrix through the recovery procedure; $S_{\mathrm{t}}^{i}$ - single cross section area of LLDPE fibril during recovery process (changing with recovery time); $l_{t}^{i}$ - single length of LLDPE fibril during recovery process (changing with recovery time); $\overline{S_{t}}-$ average cross section area of LLDPE fibrils in recovery mode; $\bar{l}_{t}$ - length of LLDPE fibril in recovery mode; $n$ number of LLDPE fibrils in recovery mode; $\lambda$ - elongational ratio in recovery mode; $\overline{l_{0}}$ - average length of LLDPE fibrils at the beginning of recovery mode; $\bar{l}_{t}$ average length of LLDPE fibrils at a certain time of recovery mode; $\overline{S_{0}}$ - average cross section area of LLDPE fibrils at the beginning of recovery mode; $\overline{S_{t}}$ - average cross section area of LLDPE fibrils at a certain time of recovery mode; $\varepsilon$ - elongational strain in recovery mode; $\frac{d \overline{S_{t}}}{d t}$ instantaneous change rate of average cross section area in recovery mode; $k$ - coefficient factor; $\eta$ - constant; $\overline{S_{\infty}}$ final average cross section area of fibrils when recovery time $t$ approaches positive infinity; $C$ - constant (intercept of fitting curve); ${\overline{S_{t}}}^{\prime}$ - first derivative of $\overline{S_{t}}$ in recovery mode; $\overline{S_{t}}{ }^{\prime \prime}$ - second derivative of $\overline{S_{t}}$ in recovery mode; $\overline{S_{t^{*}}}$ - half value of $\overline{S_{\infty}}$ in recovery mode; $t^{*}$ time when $\overline{S_{t}}$ equals $\overline{S_{t^{*}}}$ in recovery mode; $\lambda^{\prime}$ - average length of LLDPE fibrils in processing in VPE; $\varepsilon^{\prime}-$ elongational strain rate in processing in VPE.

\section{Introduction}

Over the past two decades, the production and application of polymer blends have increased enormously. It is well known that the polymer blends are the mixtures of polymers having different properties [1,2]. The performance of the polymer blends is determined by the compatibility of interface and mixing effect of polymer components $[3,4]$. Since most of the polymers are immiscible [5], the combinations of immiscible polymers involve chemical reactions and physical influences [6]. In practical, melt blending is a commonly used method to realize the intimate mixing of immiscible polymers [7].

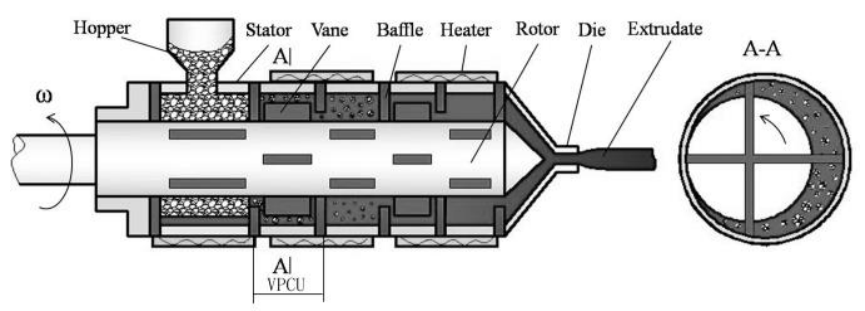

Fig. 1 Structure schematic diagram of VPE [8]

With regards to the devices used for melt blending, researchers have always used screw extruders to process polymer materials. In the current work, a Vane Plasticating Extruder(VPE), which was invented by Qu, is used [8]. of which the mechanism is nearly the same as pump in different field [9]. It has shown a number of advantages, such as adaptability for materials, low energy consumption, good dispersion effect of nanoparticles, and better droplet breakup effect, and so on. The processing mechanism of the VPE is mainly based on the "elongational rheology". However, in order to optimize the design of VPE, the processing mechanism needed to be demonstrated, and the elongational effect should be quantified. Fig. 1 shows 
the vertical cross section structure of the VPE device.

According to Fig. 1, the A-A profile shows the cross-sectional schematic of the VPE. Nineteen groups of vane plasticating and conveying units (VPCUs) constitute the main structure of the VPE. In the VPCU, a space with certain geometric shape is made up of rotors, stators, vanes of certain number, and plates for material-proofing. As the rotor is eccentric to the internal cavity of the stator, the volume would first increase and then decrease, and this trend would keep on happening successively. Polymer melts were extruded into the space through inlet, while the volume swelled. Moreover, materials are grinded, compacted, and exhausted by forces of flow field [10]. The processing can also be seen as a cyclical dynamic plasticating conveying procedure.

In order to investigate the mechanism of generation of elongational flow field by VPE, the inner structures of VPE and the morphologies of polymer melts were analysed using mathematical modelling. Furthermore, the im miscible linear low density polyethylene (LLDPE)/ polystyrene (PS) blends were extruded using the VPE, and after deformation in VPE, the evolution of phase structure was studied during the recovery mode. The morphologies of recovered materials were studied using scanning electron microscopy (SEM) and image analysis. Moreover, according to the modelling and verification, the rate of elongational deformation of LLDPE was calculated, which was used to quantify the effect of VPE.

\section{Mechanism for the verification experiment}

Though it has been proven that the VPE has a number of advantages, it is hard to measure the elongational strain rate of blends, which were directly affected by the VPE. In order to acquire more information on it, a mechanism for the deformation of blends was built. An experiment was designed to verify the mechanism.

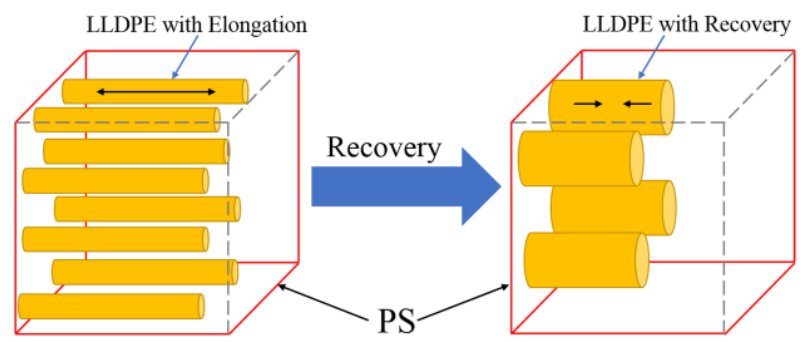

Fig. 1 Schematic diagrams of LLDPE in recovery mode after processing via VPE

According to Stary [11], the deformation of immiscible PS/LLDPE blends would recover to its relative original phase in a heated oil bath. The elongational deformation can also be expected in the VPE, both in the continuous phase PS and the dispersed phase LLDPE, which have a constant volume ratio during the recovery phase. Furthermore, due to the low content of LLDPE, the volume of the LLDPE can be considered constant. Besides, the LLDPE fibrils observed in the cross section are all treated as complete cylinders. Besides, the LLDPE fibrils observed in the cross section are all treated as complete cylinders. Therefore, the processing of stretched LLDPE fi- brils in a recovery mode was shown in Fig. 2. The volume of LLDPE ( $V_{P E}$ ) can be expressed using Eq. (1):

$$
V_{P E}=\sum_{i=1}^{n} S_{t}^{i} l_{t}^{i} \quad(i=1,2,3 \cdots n ; t \in[0,+\infty)),
$$

where: $S_{t}^{i}$ is the cross-section area and $l_{t}^{i}$ is the length of any single LLDPE fibril during recovery which changes with recovery time. However, $V_{P E}$ remains the same both in plasticating extruding and recovery phases.

$\overline{S_{t}}$ and $\overline{l_{t}}$ are the average cross-sectional area and average length of all the LLDPE fibrils and $n$ is the number of LLDPE fibrils during recovery. These can be represented using Equations (2) and (3).

$$
\begin{aligned}
& \overline{S_{t}}=\frac{\sum_{i=1}^{n} S_{t}^{i}}{n} \quad(i=1,2,3 \cdots n ; t \in[0,+\infty)), \\
& \overline{l_{t}}=\frac{\sum_{i=1}^{n} l_{t}^{i}}{n} \quad(i=1,2,3 \cdots n ; t \in[0,+\infty)) .
\end{aligned}
$$

The elongational ratio $\lambda$, and the elongational strain $\varepsilon$ during recovery can be using Eqs. (4) and (5):

$$
\begin{aligned}
& \lambda=\frac{\overline{l_{t}}}{\overline{l_{0}}} \times 100 \%=\frac{\overline{S_{0}}}{\overline{S_{t}}} \times 100 \%, \\
& \varepsilon=\frac{\overline{l_{t}}-\overline{l_{0}}}{\overline{l_{0}}}=\lambda-1,
\end{aligned}
$$

where: $\overline{l_{0}}$ is the average length and $\overline{S_{0}}$ is the average cross-sectional area of all LLDPE fibrils at the beginning of recovery phase. Additionally, $\bar{l}_{t}$ and $\overline{S_{t}}$ are the average length and the average cross-sectional area of all LLDPE fibrils at a certain time of recovery.

In the mechanical relaxation mode, mode (i.e. under real extruding conditions), the elongational ratio $\lambda^{\prime}$, and the elongational strain $\varepsilon^{\prime}$ can be expressed using Eqs. (6) and (7):

$$
\begin{aligned}
& \lambda^{\prime}=\frac{\overline{l_{0}}}{\overline{\bar{l}}} \times 100 \%=\frac{\overline{S_{t}}}{\overline{S_{0}}} \times 100 \%, \\
& \varepsilon^{\prime}=\frac{\overline{l_{0}}-\overline{l_{t}}}{\overline{l_{t}}}=\lambda^{\prime}-1,
\end{aligned}
$$


Eqs. (4-6), and (7) constitute the deformation mechanism of the blends. Later, an experiment is designed to verify the mechanism. The details about the experiment are given as follows.

\section{Experimental}

\subsection{Materials and devices}

LLDPE Q/SH 1095 5-2003 (Petroleum \& Chemical Co., Maoming, China), and PS GPPS-525 (Petroleum \& Xinzhongmei Chemical Co., Zhanjiang, China) were used as the dispersed phase and continuous phase during extruding, respectively. Castor oil CP (Damao Chemical Reagent Factory, Tianjin, China) was used as bath oil for recovery. Analytically pure ethanol (LiQiang Chemical Plant, Guangzhou, China) was used in the experiment. There were 19 VPCUs in the VPE. In one VPCU, the rotor diameter is $0.04 \mathrm{~m}$, the inner diameter of eccentric stator was $0.046 \mathrm{~m}$, the eccentricity was $0.003 \mathrm{~m}$. In order to control the temperature, a constant temperature oil bath DF-101S (Yuhua Instrument Co., Ltd., Gongyi, China) was used in the experiment.

\subsection{Preparation of specimens}

After removing moisture in a drying oven for 40 minutes at $60^{\circ} \mathrm{C}, 15 \mathrm{wt} \%$ LLDPE and $85 \mathrm{wt} \%$ PS were mixed together in a high-speed mixer for 10 minutes. To ensure that the LLDPE dispersed evenly in the PS matrix, the LLDPE/PS blends were extruded using VPE, which was developed in our own laboratory. The rotational speed was set at $60 \mathrm{r} / \mathrm{min}$. The four processing temperatures from feeding section to extrusion section were set at $150^{\circ} \mathrm{C}$, $170^{\circ} \mathrm{C}, 170^{\circ} \mathrm{C}$, and $170^{\circ} \mathrm{C}$. Then, the extruded blends were granulated using a granulator, and the pellets of blends were placed in the drying oven for another 40 minutes at $60^{\circ} \mathrm{C}$. Afterwards, the pellets of LLDPE/PS blends were extruded using the VPE at the same rotational speed and processing temperatures as before. While the extrusion was steady, specimens close to the extruding die were cut rapidly, and were immersed in the castor oil bath to recover for $0,15,30,40,50,80,120$, and 180 seconds at a constant temperature of $170^{\circ} \mathrm{C}$. Afterwards, all of the specimens were taken out and were immediately frozen in liquid nitrogen for 5 minutes to maintain the morphologies. All of them were brittle fractured in liquid nitrogen to expose the internal structures. Later, they were immersed into ethanol to remove castor oil.

\subsection{Scanning electron microscopy}

Morphological observations were made using an S-3700N (Japan) scanning electron microscope. The SEM analysis was done only for the cross-and vertical-sections only for specimens without castor oil bath treatment) of LLDPE/PS blends. Additionally, the surfaces of all the specimens were sputtered twice with gold.

\subsection{Image analysis}

The micrographs were evaluated using image analyses software (image pro plus). Image analyses consisted of counting the number of LLDPE fibrils, and measuring the diameters of the LLDPE fibrils.

\section{Results and discussion}

The SEM morphologies of the cross sections and the vertical sections of LLDPE fibrils in PS matrix without recovery are shown in Fig. 3.

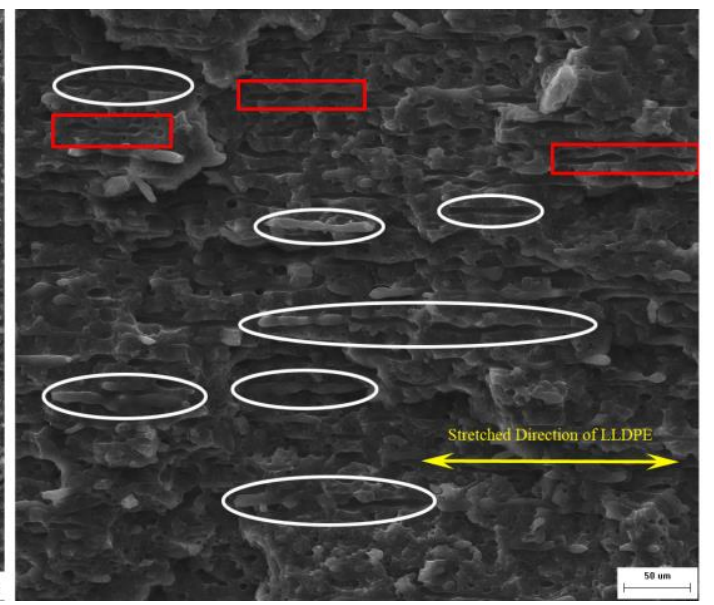

b

Fig. 3 SEM morphologies of LLDPE fibrils in PS matrix without recovery: $a$ - cross section, $b$ - vertical section

Fig. 3 (a) is the cross-sectional morphology of the LLDPE/PS blends. The LLDPE fibrils are evenly dispersed in the PS matrix, and the boundary between LLDPE and PS can be seen clearly. This was a sea-island structure of immiscible blends. The average radius and area of
LLDPE fibrils were $2.77 \times 10^{-6} \mathrm{~m}$ and $2.410 \times 10^{-11} \mathrm{~m}^{2}$, respectively. Fig. 3 (b) shows the morphology of vertical section of LLDPE fibrils in the PS matrix without recovery. The arrow in Fig. 3 (b) indicates the direction of the LLDPE particles stretched in the VPE (which is also the 
direction of elongational flow field). The LLDPE particles developed into fibrils with large aspect ratio after the elongational deformation. Several representative fibrils were marked in ellipses with white color. These LLDPE fibrils were completely exposed in the vertical section, while others were inserted or partly inserted in the PS matrix. Some LLDPE melts underwent fracturing process, while others did not, which is highlighted in red rectangles in Fig. 3 (b). Fig. 3 revealed the pattern of the stretching of
LLDPE in the elongational flow field, whereas it also presented the dispersion conditions of LLDPE in the PS matrix. In Fig. 3, the structure of most LLDPE fibrils were approximated to be cylindrical, which indicated that Eqs. (6) and (7) were suitable for calculating the elongational ratio $\lambda^{\prime}$ and the elongational strain $\varepsilon^{\prime}$ of melts which were processed by the VPE.
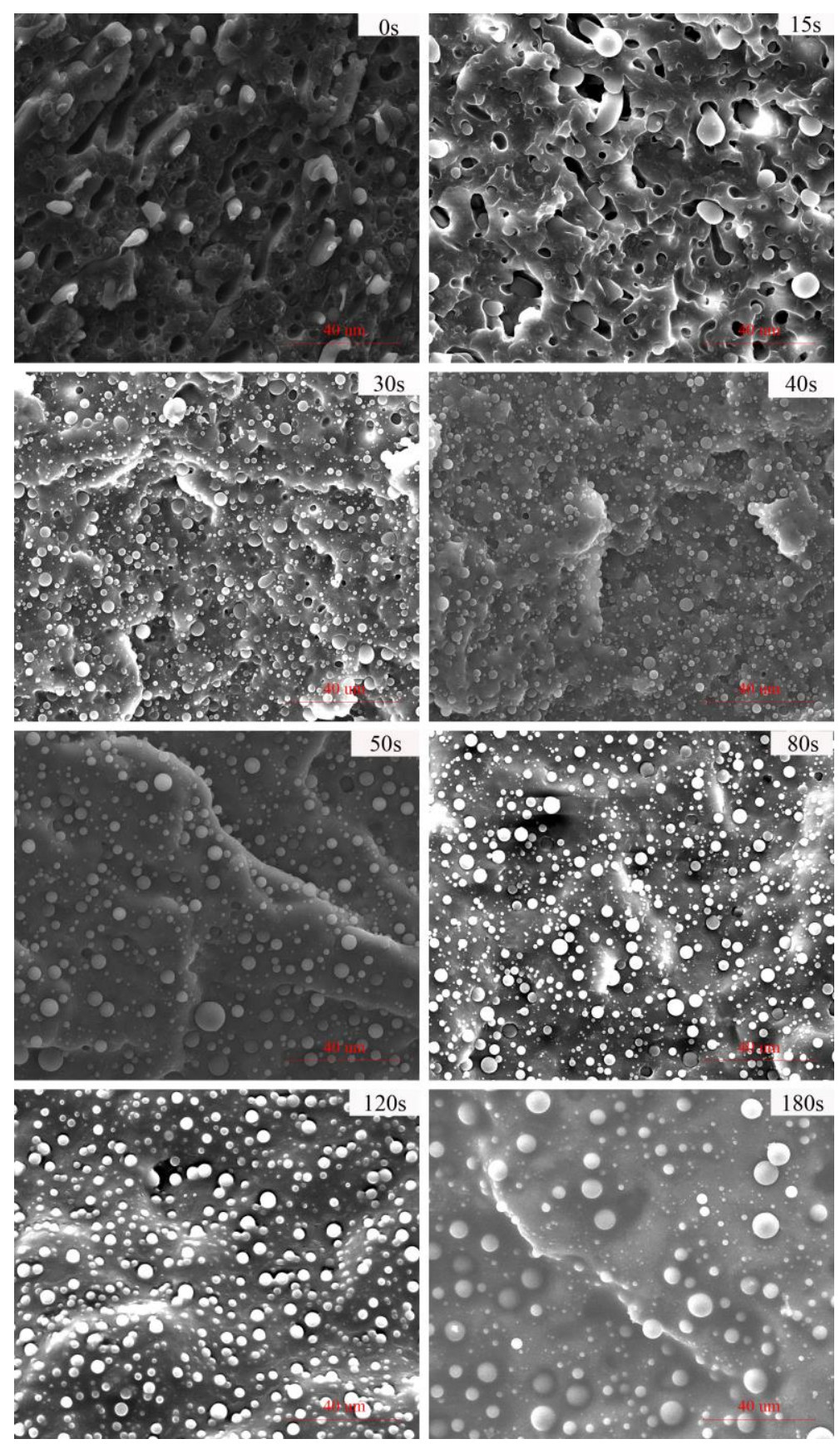

Fig. 4 Morphology development during recovery

The cross-sectional morphology development of LLDPE is presented in Fig. 3 and Fig. 4. The cross-sectional morphology was a sea-island structure for the recovery time of $0 \mathrm{sec}$. However, several cross sections of LLDPE fibrils were shown as ellipses in Fig. 4 (0s), which implied that the elongational effect was not only in the axial direction, but was also present along the circumference. The elongational effect on fibrils along the cir- cumference might have happened during the transfer of melt from one VPCU to another. It also proved that the effect along the circumference was weaker than that in the axial direction. According to the statistical analysis shown in Fig. 3 (b) (40 fibrils of LLDPE), the average length of LLDPE fibrils along the axial direction was $6.82 \times 10^{-5} \mathrm{~m}$, and the average diameter was $4.93 \times 10^{-6} \mathrm{~m}$. Thus, the 
aspect ratio was 13.83, which meant that the LLDPE particles were stretched into fibrils due to the processing of the VPE.

The morphology started to change when the recovery time was $15 \mathrm{sec}$. As can be seen in Fig. 4 (15 sec), some of the sea-island structures survived, while others became continuous phase. It is also from Fig. $4(30 \mathrm{sec})$ that when the specimens were immersed in oil bath for 30 sec, the stress between LLDPE and PS decreased. Additionally, the cross section of LLDPE recovered to a regular sea-island structure, and became evenly dispersed in the PS matrix. When the recovery time was within [30 sec, 80 sec], the average radius of LLDPE cross section increased steadily, and this increase was reflected in Fig. 4 (30 sec), $(40 \mathrm{sec}),(50 \mathrm{sec}),(80 \mathrm{sec}),(120 \mathrm{sec}),(180 \mathrm{sec})$, and Fig. 5. As can be seen from Fig. $4(120 \mathrm{sec})$, LLDPE was well distributed in the PS matrix, while the cross section of LLDPE fibrils was the most homogeneous in all the specimens. Finally, Fig. $4(180 \mathrm{sec})$ showed the cross-sectional morphology of LLDPE fibrils, which started to change again. This might be due to the sustainable heating, which made some LLDPE fibrils to further move and merge together.

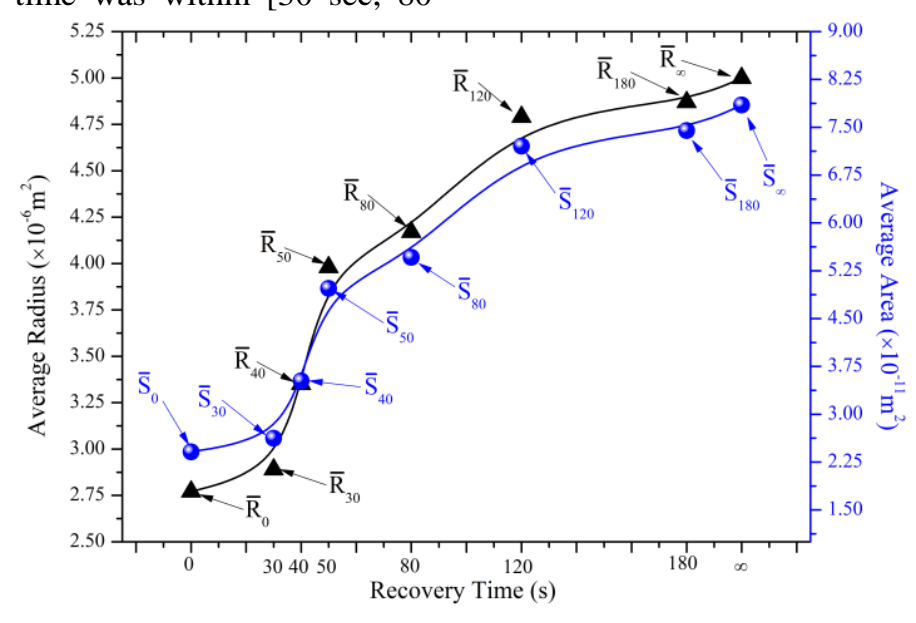

Fig. 5 Average cross section radius and area of LLDPE fibrils in PS matrix

Based on the statistical means of "image pro plus" software, the cross section of LLDPE fibrils with different recovery times were analyzed. The values of average radiuses and areas were shown in Fig. 5 and Table 1. The values were the smallest when the recovery time was $0 \mathrm{sec}$, while both of them increased with the extension of the recovery time. The values reached the maximum when the recovery time was $180 \mathrm{sec}$, The maximum values for radius and area were $4.87 \times 10^{-6} \mathrm{~m}$, and $7.447 \times 10^{-11} \mathrm{~m}^{2}$, respectively.

Table 1

Average cross-sectional radius and area of LLDPE fibrils

\begin{tabular}{|c|c|c|}
\hline $\begin{array}{c}\text { Recovery } \\
\text { Time }(\mathrm{s})\end{array}$ & $\begin{array}{c}\text { Average cross section } \\
\text { radius }(\mathrm{m})\end{array}$ & $\begin{array}{c}\text { Average cross section area } \\
\left(\mathrm{m}^{2}\right)\end{array}$ \\
\hline 0 & $2.77 \times 10^{-6}$ & $2.409 \times 10^{-11}$ \\
\hline 15 & - & - \\
\hline 30 & $2.89 \times 10^{-6}$ & $2.623 \times 10^{-11}$ \\
\hline 40 & $3.35 \times 10^{-6}$ & $3.524 \times 10^{-11}$ \\
\hline 50 & $3.98 \times 10^{-6}$ & $4.974 \times 10^{-11}$ \\
\hline 80 & $4.17 \times 10^{-6}$ & $5.460 \times 10^{-11}$ \\
\hline 120 & $4.79 \times 10^{-6}$ & $7.204 \times 10^{-11}$ \\
\hline 180 & $4.87 \times 10^{-6}$ & $7.447 \times 10^{-11}$ \\
\hline
\end{tabular}

According to the visual curve tendency (as shown in Fig. 5) and the data from Table 1, the variation in the cross section of LLDPE could be divided into two sections. In one section, the LLDPE/PS melt blends show a rapidly changing morphology for the recovery time of [0 sec, 15 sec]. The melt was shown as a continuous phase in Fig. 4 (15 sec). It was difficult to distinguish between the LLDPE phase and the PS phase. Therefore, the radius of the LLDPE fibrils could not be provided in Table 1. In the other section, the cross section of LLDPE fibrils varied steadily in [15 sec, $180 \mathrm{sec}$, while the radius increased steadily and reached its maximum value. It could be inferred that the LLDPE fibrils transformed steadily. The length was shorter, and the cross-sectional area was larger. The experiment reached its end when the recovery time was $180 \mathrm{sec}$, The average value of radius was the highest among all the observations.

The fibrils gradually shrank back to original phase, however, the average cross-sectional area of fibrils increased with restrictions. Therefore, a recovery model should be developed, according to which, the length and diameter of fibrils can be predicted at any time.

In the light of existing data, the average rate of change in the cross-sectional area of LLDPE fibrils between time range $\Delta t$ is defined as:

$$
\frac{\Delta \overline{S_{t}}}{\Delta t}=\frac{\bar{S}_{(t+\Delta t)}-\bar{S}_{t}}{\Delta t}=k \overline{S_{t}} \Rightarrow \lim _{\Delta t \rightarrow 0}=\frac{\Delta \overline{S_{t}}}{\Delta t}=\frac{d \overline{S_{t}}}{d t}=k \overline{S_{t}} ; t \in[0,+\infty),
$$

where: $\frac{d \overline{S_{t}}}{d t}$ is the instantaneous rate of change of aver- age cross-sectional area and $k$ is the coefficient factor, which can be expressed as: 


$$
k=\eta\left(\overline{S_{\infty}}-\overline{S_{t}}\right)
$$

In Eq. (14), $\eta$ is a constant, $\overline{S_{\infty}}$ is the average cross-sectional area of LLDPE fibrils when the recovery time $t$ approaches positive infinity. Eqs. (8) and (9) can be used to get:

$$
\frac{d \overline{S_{t}}}{d t}=\eta\left(\overline{S_{\infty}}-\overline{S_{t}}\right) \overline{S_{t}}
$$

After deformation, Eq. (10) changes to:

$$
\frac{d \overline{S_{t}}}{\overline{S_{t}}}+\frac{d \overline{S_{t}}}{\overline{S_{\infty}}-\overline{S_{t}}}=\eta \overline{S_{\infty}} d t
$$

After integrating Eq. (11), Eq. (12) is obtained as:

$$
\ln \frac{\overline{S_{t}}}{\overline{S_{\infty}}-\overline{S_{t}}}=\eta \overline{S_{\infty}} t+C
$$

Assuming that $\overline{S_{t}}<\overline{S_{\infty}}$ and taking $\overline{S_{t}}<\overline{S_{0}}$, constant $C$ could be calculated as:

$$
C=\ln \frac{\overline{S_{0}}}{\overline{S_{\infty}}-\overline{S_{0}}}-\eta \overline{S_{\infty}} \times 0=\ln \frac{2.409 \times 10^{-11}}{\overline{S_{\infty}}-2.409 \times 10^{-11}} .
$$

Eqs. (12) and (13) are used to obtain Eq. (14).

$$
\ln \frac{\overline{S_{t}}\left(\overline{S_{\infty}}-\overline{S_{0}}\right)}{\overline{S_{0}}\left(\overline{S_{\infty}}-\overline{S_{t}}\right)}=\eta \overline{S_{\infty}}(\mathrm{t}-0)
$$

After deformation, Eq. (14) becomes:

$$
\frac{\overline{S_{t}}\left(\overline{S_{\infty}}-\overline{S_{0}}\right)}{\overline{S_{0}}\left(\overline{S_{\infty}}-\overline{S_{t}}\right)}=e^{\eta \overline{S_{\infty}}(\mathrm{t}-0)}
$$

When $t$ approaches positive infinity, the following analytical expression is obtained:

$$
\overline{S_{t}}=\frac{\overline{S_{\infty}} \overline{S_{0}}}{\left[\overline{S_{0}}+\left(\overline{S_{\infty}}-\overline{S_{0}}\right) e-\eta \overline{S_{\infty}} t\right]} .
$$

When the average cross-sectional radius of LLDPE fibrils is set to be $5.000 \times 10^{-6} \mathrm{~m}$ the value of $\overline{S_{\infty}}$ obtained is:

$$
\overline{S_{\infty}} \approx 7.850 \times 10^{-11} \mathrm{~m}^{2}
$$

The value of $\overline{S_{\infty}}$ is used in Eq. (16) to get:

$$
\ln \frac{\overline{S_{t}}}{7.850 \times 10^{-11}-\overline{S_{t}}}=7.850 \times 10^{-11} \eta t+C
$$

Combining with the experimental data, curve fitting is undertaken. It can be seen from Fig. 6 that the fitted curve is a straight line with slope $\eta \overline{S_{\infty}}$ equal to 0.024 , and the intercept $C$ of -0.996 . The equation of the fitted curve is:

$\ln \frac{\overline{S_{t}}}{\overline{S_{\infty}}-\overline{S_{t}}}=0.024 t-0.996 \Rightarrow\left\{\begin{array}{l}\eta=3.019 \times 10^{8} \\ C=-0.996\end{array}\right.$

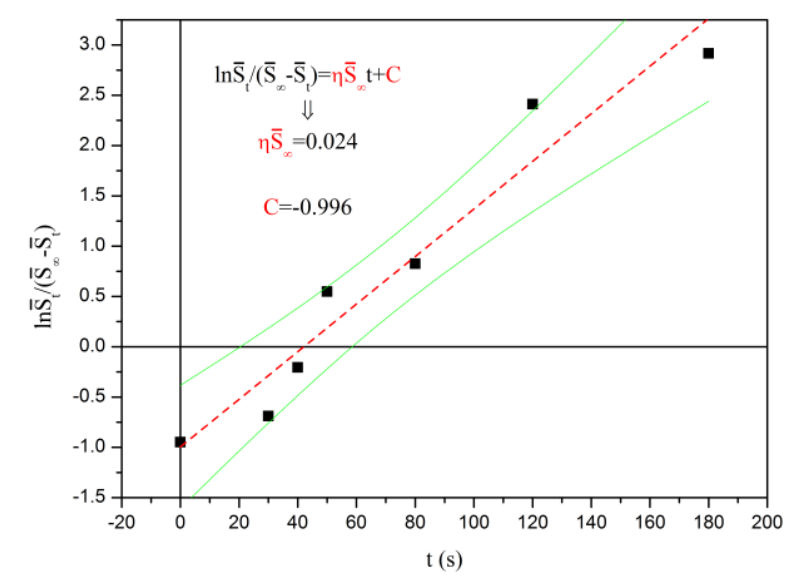

Fig. 6 Curve fitting to obtain the slope and intercept related to recovery time

According to Eq. (19), when $t$ reaches positive infinity, $\overline{S_{t}}$ approaches $\overline{S_{\infty}}$. The second derivative of $\overline{S_{t}}$ is calculated using Eq. (10):

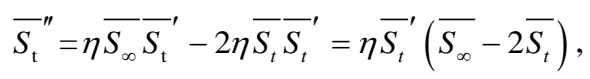

when: $\overline{S_{t}}=\frac{\overline{S_{\infty}}}{2},{\overline{S_{t}}}^{\prime \prime}=0, \frac{d \overline{S_{t}}}{d t}$ approaches the maximum value. In other words, the instantaneous rate of change of average cross-sectional area becomes maximum.

$$
\overline{S_{t *}}=\frac{\overline{S_{\infty}}}{2}=3.925 \times 10^{-11} \mathrm{~m}^{2}
$$

where: $\overline{S_{t^{*}}}$ is the half value of $\overline{S_{\infty}}$ during recovery. Using Eq. (18) and Eq. (21), Eq. (22) can be obtained as:

$$
t^{*}=0-\frac{1}{\eta \overline{S_{\infty}}} \ln \frac{\overline{S_{0}}}{\overline{S_{\infty}}-\overline{S_{0}}}=40.001 \mathrm{~s} .
$$


By putting $t^{*}$ in Eq. (24), Eq. (28) is acquired as:

$$
\overline{S_{t}}=\frac{\overline{S_{\infty}}}{1+e^{-\eta \overline{S_{\infty}}\left(t-t^{*}\right)}}
$$

By inserting $\overline{S_{\infty}}$ and $t *$ in Eq. (23), the expression for $\overline{S_{t}}$ is acquired to be as:

$$
\overline{S_{t}}=\frac{7.85 \times 10^{-11}}{1+2.581 e^{-0.024 t}} .
$$

Fig. 7 shows the scatter diagram of measured experimental data, and the derivative functional curve having 2000 collected points. There is no sudden change of morphology during the recovery. The fibrils gradually shrink back to a certain steady state, and the measured data coincides well with the theoretical curve. From the theoretical curve, it can be seen that when the recovery time is 40.001 sec, the curve ascends with the highest slope. This means that the fibrils shrink most severely at this point in time. It can be predicted that the average cross-sectional area fluctuates only slightly in an extended time period. In other words, the fibrils are in a relatively steady state even after $180 \mathrm{sec}$.

According to the model, large amount of LLDPE fibrils would be transformed into more complete cylinders having less structural defects, while these cylinders would be dispersed uniformly under the premise that the LLDPE/PS melt is immersed in the oil bath for as long as possible.

Based on the results, it can be inferred from the statistical data that the LLDPE fibrils would be in a relatively complete cylindrical form for the recovery time of $180 \mathrm{sec}$. When the recovery time approached positive infinity, the data can be used to calculate the elongational effect that generated by SRVPE and imposed on the LLDPE/PS melt. According to the Eqs. (4) and (5), the elongational ratio $\lambda$, and the elongational strain $\varepsilon$ could be calculated using Equations (25) and (26).

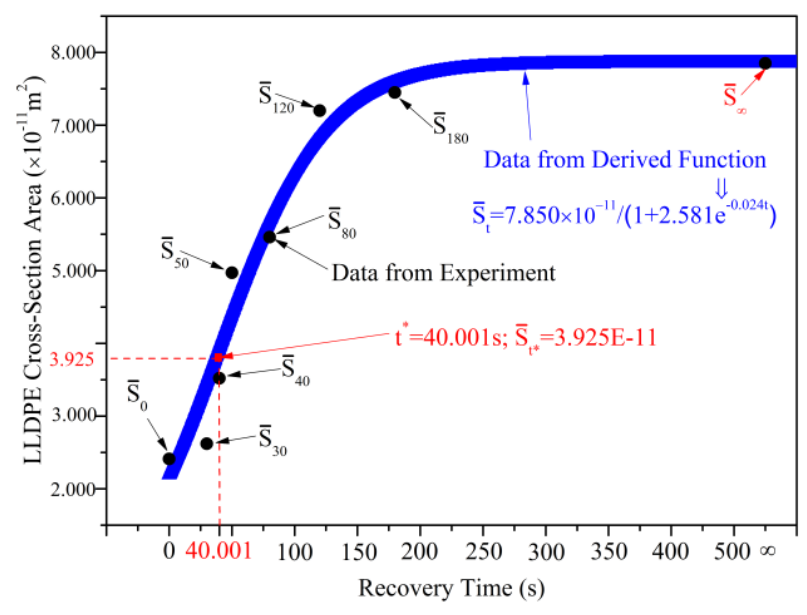

Fig. 7 Comparison between curves of derivative function and measured data

$$
\begin{aligned}
& \lambda=\frac{\overline{l_{t}}}{\overline{l_{0}}}=\frac{\overline{l_{\infty}}}{\overline{l_{0}}}=\frac{\overline{S_{0}}}{\overline{S_{t}}}=\frac{\overline{S_{0}}}{\overline{S_{\infty}}}=\frac{2.409 \times 10^{-11}}{7.850 \times 10^{-11}} \times \\
& \times 100 \%=30.688 \%
\end{aligned}
$$

$$
\varepsilon=\frac{\overline{l_{t}}-\overline{l_{0}}}{\overline{l_{0}}}=\frac{\overline{l_{\infty}}-\overline{l_{0}}}{\overline{l_{0}}}=\lambda-1=-0.693
$$

The final status of the strain of melt that was effected by the elongational flow field can be represented by Eq. (25). It means that the average length of LLDPE fibrils shrink to $30.688 \%$ of the original one, while the elongational strain decreases to 0.693 . As the controlled recovery experiment is a designed as an adverse experiment, the final status can be seen as the one where the elongational flow field began to have an effect on the fibrils via processing, while the original status remains as it is when the elongational flow field is not acting. Consequently, the average length of LLDPE fibrils increased by $325.861 \%$ (Equation (27)), while the elongational strain rate was 2.259 (Equation (28)).

$$
\begin{aligned}
& \lambda^{\prime}=\frac{\overline{l_{0}}}{\overline{l_{t}}}=\frac{\overline{l_{0}}}{\overline{\overline{l_{\infty}}}}=\frac{\overline{S_{t}}}{\overline{S_{0}}}=\frac{\overline{S_{\infty}}}{\overline{S_{0}}}=\frac{7.850 \times 10^{-11}}{2.409 \times 10^{-11}} \times \\
& \times 100 \%=325.861 \% \\
& \varepsilon^{\prime}=\frac{\overline{l_{0}}-\overline{l_{t}}}{\overline{l_{t}}}=\frac{\overline{l_{0}}-\overline{l_{\infty}}}{\overline{l_{\infty}}}=\lambda^{\prime}-1=2.259
\end{aligned}
$$

\section{Conclusions}

Based on the structural analysis, a mathematical model was proposed, and the results verified the processing mechanism in VPE. It was found that the elongational flow field did exist during the circumferential VPCU revolving in each inter cavity. The results from the recovery experiment confirmed these findings.

In the specimens without recovery, the average aspect ratio of LLDPE fibrils is 13.83. Another mathematical model was established to predict the evolution tendency of LLDPE morphology. The process of curve fitting predicted that the cross section shrinks rapidly during the recovery time of 0-180 sec, while afterwards it remained relatively stable. The fiercest morphological evolution occurred at $40.001 \mathrm{sec}$. The genuine elongational ratio $\lambda^{\prime}$, and elongational strain $\varepsilon^{\prime}$ via VPE processing were found to be $325.861 \%$ and 2.259 , respectively. These results established that the VPE does impose elongational flow field on LLDPE fibrils. The processing mechanism and elongational effect of VPE were valuable for optimizing the designing of VPE and promoting th6.e development of polymer processing theory.

\section{Acknowledgements}

This work is supported by the National Nature Science Foundation of China (51501130); Tianjin science and technology correspondent project (15JCTPJC60400); Tianjin science and technology innovation system and 
platform construction plan (14TXGCCX00011); Subject Leader Training Program of Tianjin Universities and colleges (RC14-02); 973 Program (2012CB025902); the National Research Foundation for the Doctoral Program of Higher Education of China (20120172130004); The Special-funded Program on National Key Scientific Instruments and Equipment Development of China (2012YQ230043); The National Nature Science Foundation of China (51435005); The Research Development Foundation of Tianjin University of Technology and Education (KJ11-06). Science and Technology Pre Research Project of Tianjin University of Technology and Education (KJY14-03). The Research Development Foundation of Tianjin University of Technology and Education (XJKC031420); The Research Development Foundation of Tianjin University of Technology and Education (XJKC031451). The science and technology development fund project of Tianjin University (JWK1711). The authors would like to take this opportunity to express their sincere appreciation.

\section{References}

1. Jazani, O. M.; Arefazar, A.; Beheshty, M. H. 2010. Study on the effect of processing conditions on the mechanical properties of PP/PC/SEBS ternary blends using Taguchi experimental analysis, E-Polymers 70: p. 263-275. https://doi.org/10.1515/epoly.2010.10.1.1296.

2. Ostaševičius, V.; Milašauskaitė, I.; Daukševičius, R.; Baltrušaities, V.; Grigaliūnas, V.; Prosyčevas. 2010. Experimental characterization of material structure of piezoelectric PVDF polymer, Mechanika 6(86): p.78.

3. Poyekar, A. V.; Bhattacharyya, A. R., Panwar, A. S.; Simon, G. P.; Sutar, D. S. 2014. Influence of noncovalent modification on dispersion state of multiwalled carbon nanotubes in melt-mixed immiscible polymer blends, Applied materials \& interfaces 6(14): p.11054-11067. https://doi.org/10.1021/am501737z.

4. Dimzoski, B.; Fortelný, I.; Šlouf, M.; Sikora, A.; Michálková, D. 2013. Morphology evolution during cooling of quiescent immiscible polymer blends: matrix crystallization effect on the dispersed phase coalescence, Polymer Bulletin 70(1): p.263-275. https://doi.org/10.1007/s00289-012-0848-1.

5. Göldel, A.; Kasaliwal, G. R.; Pötschke, P.; Heinrich, G. 2012. The kinetics of CNT transfer between immiscible blend phases during melt mixing, Polymer 53(2): p.411-421. https://doi.org/10.1016/j.p olymer.2011.11.039.

6. Dabkevičius, A.; Kibirkštis, E. 2006. Investigation of mechanical characteristics of polymer films for packaging production, Mechanika 4(60): p.5.
7. Orihara, H.; Nishimoto, Y.; Aida, K.; Na, Y. H. 2011. Three-dimensional observation of an immiscible polymer blend subjected to a step electric field under shear flow, Physical Review 83: p.026302. https://doi.org/10.1103/physreve.83.026302.

8. Qu, J. P.; Zhang, G. Z.; Chen, H. Z.; Yin, X. C.; He, H. Z. 2012. Solid conveying in vane extruder for polymer processing: Effects on pressure establishment, Polymer Engineering \& Science 52(10): p.2148. https://doi.org/10.1002/pen.23176.

9. Allali, A.; Belbachir, S.; Lousdad, A. 2016. Finite element based design of a polymer rotor of centrifugal pump, Mechanika 22(1): p.38. https://doi.org/10.5755/j01.mech.22.1.12218.

10. Qu, J. P.; Zhao, X. Q.; Li, J. B.; Cai, S. Q. 2013. Power consumption in the compacting process of polymer particulate solids in a vane extruder, Journal of applied polymer science 127(5): p.3923-3932. https://doi.org/10.1002/app.36806

11. Stary. Z.; Munstedt, H. 2008. Morphology Development in PS/LLDPE blend during and after elongational deformation, Journal of Polymer Science: Part B Polymer Physics 46(1): p.16-27.

https://doi.org/10.1002/polb.21338.

Zhenghuan Wu, Quan Wang, Yongqing Zhao, Qixiang Fan, Hai Yang, Jinping Qu

\section{ELONGATIONAL EFFECT ON IMMISCIBLE POLYMER BLENDS VIA NOVEL VANE PLASTICATING EXTRUDER}

S u m m a r y

The vane plasticating extruder (VPE) is a novel polymer processing equipment which is based upon elongational rheology. According to the basic structural analysis and movement interpretation, generation mechanism of elongational flow field was derived. Elongational effects during the controlled recovery of immiscible polymer blends, containing polystyrene (PS) matrix and linear low density polyethylene (LLDPE) as dispersed phase, were studied. The morphological results revealed the elongational effect of vane extruder. LLDPE morphology evolution was precisely predicted by a mathematical model. Elongational ratio and elongational strain via VPE were calculated separately, both of which established that the VPE does imposed elongational force field on polymer blends.

Keywords: vane plasticating extruder; elongational flow field; controlled recovery; mechanism.

Received December 09, 2016 Accepted December 07, 2017 